Abstract

\title{
A New Beamline for Advanced Photoelectron Spectroscopy Based on Extreme Ultraviolet High Harmonics at High Repetition Rate ${ }^{\dagger}$
}

\author{
Riccardo Cucini 1,*, Tommaso Pincelli 1, Giancarlo Panaccione 1, Damir Kopic 2, Fabio Frassetto 3, \\ Paolo Miotti ${ }^{3,4}$, Gian Marco Pierantozzi ${ }^{1}$, Simone Peli ${ }^{2}$, Andrea Fondacaro ${ }^{1}$, \\ Aleksander De Luisa ${ }^{1}$, Alessandro De Vita ${ }^{5}$, Damjan Krizmancic ${ }^{1}$, Daniel T. Payne ${ }^{2}$, \\ Federico Salvador ${ }^{1}$, Andrea Sterzi ${ }^{2}$, Luca Poletto ${ }^{3}$, Fulvio Parmigiani 2,6,7, Giorgio Rossi ${ }^{1,5}$ and \\ Federico Cilento $^{2}$ \\ 1 C.N.R.-I.O.M., Strada Statale 14, km 163.5, 34149 Trieste, Italy \\ 2 Elettra-Sincrotrone Trieste S.C.p.A., Strada Statale 14, km 163.5, 34149 Trieste, Italy \\ 3 IFN-CNR, Via Trasea 7, 35131 Padova, Italy \\ 4 Dipartimento di Ingegneria dell'Informazione, Università di Padova, via Gradenigo 6/B, \\ 35131 Padova, Italy \\ 5 Dipartimento di Fisica, Università di Milano, via Celoria 16, 20133 Milano, Italy \\ 6 Università degli Studi di Trieste, Via A. Valerio 2, 34127 Trieste, Italy \\ 7 International Faculty, University of Cologne, Albertus-Magnus-Platz, 50923 Cologne, Germany \\ * Correspondence: cucini@iom.cnr.it \\ + Presented at the 37th International Symposium on Dynamical Properties of Solids (DyProSo 2019), Ferrara, \\ Italy, 8-12 September 2019.
}

Published: 5 September 2019

Spectroscopy in the femtosecond time domain can both reveal fundamental insight in the properties of materials and provide relevant experimental tests for functional systems. The quest for sources of ultrashort photon pulses ( $100 \mathrm{fs}$ ) in the Extreme Ultraviolet (EUV) region operating with an adjustable repetition rate up to the $\mathrm{MHz}$ range has led, in this last years, to the development of high harmonic generation (HHG) coherent sources based on table-top lasers. In particular, a comprehensive characterization of the photoelectron final state in ordered solids requires measurement, with a sub- picosecond time resolution, of energy, momentum and spin-polarization of the photo-emitted current, with an energy and a momentum resolution comparable to those achieved using advanced synchrotron radiation sources.

We have built and characterized a versatile twin-beamline for advanced photoemission experiments based on a table-top laser HHG source operating in the EUV range up to $200 \mathrm{kHz}$ repetition rate. The beamline is able to provide $10^{12}$ photons/sec in the range $15-35 \mathrm{eV}$, and $10^{9}$ photons/sec up to $75 \mathrm{eV}$, with variable repetition rate, allowing one to measure shallow core level photoemission spectra in combination with valence band ones.

Experiments on metal and topological insulator have been performed to test the capabilities of the beamline, showing a pulse duration shorter than $100 \mathrm{fs}$ and an energy resolution lower than 35 $\mathrm{meV}$. The possibility to choose the optimal repetition rate for a given experiment (up to at least 200 $\mathrm{kHz}$, or lower) makes it possible to explore a wide excitation-fluence range in pump-probe experiments, minimizing sample heating when relevant.

The twin-branch beamline operates as a user's facility. It represents a unique instrument for dynamical "all resolved photoemission experiments" to study excited states and transient electronic and magnetic configurations at surfaces, nanostructures and solids, with the possibility of covering the full Brillouin zone of complex materials. 
(C) 2019 by the authors. Licensee MDPI, Basel, Switzerland. This article is an open access article distributed under the terms and conditions of the Creative Commons Attribution (CC BY) license (http://creativecommons.org/licenses/by/4.0/). 